\title{
DNA barcoding of blackflies (Diptera: Simuliidae) as a tool for species identification and detection of hidden diversity in the eastern regions of Spain
}

Ignacio Ruiz-Arrondo ${ }^{1,2^{*}}$, Luis M. Hernández-Triana ${ }^{3^{*}}$, Aleksandra Ignjatović-Ćupina ${ }^{4}$, Nadya Nikolova ${ }^{5}$, Javier Alfonso Garza-Hernández ${ }^{6}$, Mario Alberto Rodríguez-Pérez ${ }^{7}$, José A. Oteo ${ }^{1}$, Anthony R. Fooks ${ }^{3,8}$ and Javier Lucientes Curdi ${ }^{2}$

\begin{abstract}
Background: Blackflies have negative impact on public and animal health due to the haematophagous habit of females. In recent times, in some regions in Spain, blackfly outbreaks are becoming more and more frequent, threatening the public health. However, there is still a paucity of data concerning the Spanish blackfly fauna. Correct identification of species is of paramount importance in order to provide correct information on species distribution, biology and behaviour, so that control measures could be implemented appropriately.

Methods: Blackflies specimens (larvae, pupae, reared adults and biting females) were collected in the period 2015-2017 in and near rivers and streams from different regions in Spain. A modified Hotshot technique was used for the DNA extraction and the cox 1 DNA barcoding region of the cytochrome $c$ oxidase subunit 1 was sequenced from the specimens collected.
\end{abstract}

Results: In total, we collected 239 specimens representing 22 species. Of these, six species are new records for the Aragón region: P. tomosvaryi, S. bertrandi, S. galloprovinciale, S. lineatum, S. rubzovianum and S. xanthinum. Cox1 DNA barcode sequences for 21 species were recovered, including four species of the genus Prosimulium and 17 species of the genus Simulium [Boophthora (1 species), Eusimulium (1 species), Nevermannia (4 species), Simulium (s.s.) (6 species), Trichodagmia (1 species) and Wilhelmia (4 species)]. For the first time the complete DNA barcodes for five species (P. tomosvaryi, S. carthusiense, S. brevidens, S. monticola and S. sergenti) were registered. Most of the specimens belonging to the same recognized species were clustered together in the neighbour-joining tree, except for S. argyreatum, S. monticola and S. variegatum. The overall genetic distance in the dataset was $0.14 \%$. The average of the intraspecific genetic divergence within the different taxa was $1.47 \%(0.05-3.96 \%)$. In contrast, the interspecific divergence varied between $2.50-22.0 \%$.

Conclusions: In this study we assessed the use of the cox 1 DNA barcoding region for the identification of species of blackflies in Spain. Our results showed that combining DNA barcoding with morphology enhanced our taxonomic rationale in identifying the blackflies in the country.

Keywords: DNA barcoding, Blackfly, Simuliidae, Cryptic diversity, Genetic distance, New records, Aragón region, Spain

\footnotetext{
* Correspondence: irarrondo@riojasalud.es; luis.hernandez-

triana@apha.gsi.gov.uk

${ }^{1}$ Center for Rickettsiosis and Arthropod-Borne Diseases, Hospital Universitario

San Pedro-CIBIR, Logroño, La Rioja, Spain

${ }^{3}$ Wildlife Zoonoses and Vector-borne Diseases Research Group, Virology

Department, Animal and Plant Health Agency, Addlestone, UK

Full list of author information is available at the end of the article
}

(c) The Author(s). 2018 Open Access This article is distributed under the terms of the Creative Commons Attribution 4.0 International License (http://creativecommons.org/licenses/by/4.0/), which permits unrestricted use, distribution, and reproduction in any medium, provided you give appropriate credit to the original author(s) and the source, provide a link to the Creative Commons license, and indicate if changes were made. The Creative Commons Public Domain Dedication waiver (http://creativecommons.org/publicdomain/zero/1.0/) applies to the data made available in this article, unless otherwise stated. 


\section{Background}

The family Simuliidae (Diptera) includes 26 genera and 2351 species (2335 extant and 16 fossil) [1]. The females of many blackfly species bite humans, birds and other animals due to their need of blood for full egg development $[2,3]$. As a consequence of this hematophagic habit, simuliids can act as intermediate hosts of pathogens affecting the health of humans and animals worldwide [4]. In addition, blackflies can be used as water quality indicators due to the ecological demands and the role that the larvae play in rivers [5-9].

Integrated taxonomic research on the European blackfly fauna has been intensified in recent years, as demonstrated by the studies by Day [10], Day et al. [11, 12], Ilmonen et al. [13], Kúdela et al. [14, 15] and Adler et al. [16]. However, there is still a paucity of data concerning the blackfly fauna of Spain, where populations of some pest species have expanded recently and have become an emerging public and veterinary concern $[17,18]$. In the city of Zaragoza with 700,000 inhabitants, public and animal health problems are evident because of the abundance of biting blackflies, which have resulted in a serious discomfort of herds of sheep and horses and an increase of more than 200\% in recorded bites to humans between 2011 and 2012 [17, 19, 20].

Because of their environmental importance together with their impact on public and animal health, the correct identification of this insect group is of a fundamental importance in order to provide correct information on species distribution, biology and behaviour, so that targeted control measures could be correctly applied. However, standard method to blackfly species identification are mainly based on morphology, which typically require expert knowledge and sometimes the resolution can be poor because of the presence of hidden diversity [3, 21-24]. In this study we developed a molecular platform based on the cox 1 DNA barcoding region in order to support the species identification of the poorly-studied blackfly fauna of Spain. Additionally, we explored the barcode variability to reveal hidden diversity by comparing the intra- and interspecific genetic divergence within the species we analyzed.

\section{Methods}

\section{Source of material and morphological identification}

Collecting protocols established by Hernández-Triana [25] were used to collect blackflies. Larvae, pupae and link-reared adults were collected in the period 20152017 in rivers and streams across the Aragón region, although other areas were also surveyed (Fig. 1). Efforts were also made to collect females of species known to bite humans or livestock. Pre-imaginal specimens were preserved in $95 \%$ ethanol and held at $5{ }^{\circ} \mathrm{C}$ until molecular analysis. Adult specimens were preserved dry at $-40{ }^{\circ} \mathrm{C}$. Morphological identification of the collected material was based on descriptions given in identification keys of González [26], Bass [27] and Rivosecchi et al. [28].

\section{DNA extraction, PCR and sequencing}

The protocols of Hernández-Triana et al. [3, 24] were used to carry out all molecular work. When pupae were selected for analysis, most of the thorax, gill, and cocoon were retained as a voucher, while the pupal abdomen and/ or the thoracic region (ventral side) were used for DNA extraction. In the case of adults, only leg(s) were removed from the specimen for DNA extraction, while the remainder of the specimen was retained as a voucher. In the case of larvae, a thin layer of the integument was removed as a tissue source for analysis, while the remainder of the specimen was kept for further morphological examination.

A modified Hotshot technique from Montero-Pau et al. [29] was used for the DNA extraction. In brief, tissues were put directly into $50 \mu \mathrm{l}$ of alkaline lysis buffer in a 96 well-plate, and sonicated in a water bath for $20 \mathrm{~min}$. Subsequently, the plate was incubated in a PCR block machine for $30 \mathrm{~min}$ at $94{ }^{\circ} \mathrm{C}$, and allowed to cool down for $5 \mathrm{~min}$ at $4{ }^{\circ} \mathrm{C}$. Then, the plate was centrifuged for 3 min at $3000 \times \mathrm{rpm}$ after which $50 \mu \mathrm{l}$ of the neutralizing buffer was added. The plate was centrifuged again for 10 min at $3000 \times \mathrm{rpm}$ and stored at $-80{ }^{\circ} \mathrm{C}$ until processing the following day.

Detailed specimen records and sequence information (including trace files) were uploaded to the Barcode of Life Database (BOLD) (see http://www.boldsystems.org) and can be found within the Working Group 1.4 Initiative Human Pathogens and Zoonoses. The Digital Object Identifier (DOI) for the BOLD project is: dx.doi.org/10.5883/DS-SPSIM. All sequences have also been submitted to the GenBank database under the accession numbers MG894170-MG894340). Detailed record information can be found in the following projects in BOLD: "DNA barcoding Spanish Blackflies [SPSIM, SPSIB, SIMSP]".

PCR amplification was carried out using the Folmer primers [30] (LCO1490 and HCO2198), which are considered the standard for the amplification of the $658 \mathrm{bp}$ region located at the $5^{\prime}$ end of the cox 1 gene $[31,32]$. PCR products were obtained using a QIAgen PCR system using the protocol of Hernández-Triana et al. [22]. We run a $1.5 \%$ agarose gel to visualize the PCR products and samples showing correct band size were sequenced in both directions using the ABI PRISM ${ }^{\circ}$ BigDye ${ }^{\oplus}$ Terminator v3.1 Cycle Sequencing Kit (Applied Biosystems) at the Sequencing Unit, Animal and Plant Health Agency, UK.

\section{Sequence analysis}

All bi-directional sequences were combined to produce a single consensus sequence, the full length 658 bp barcode. For certain taxonomically problematic species, the 


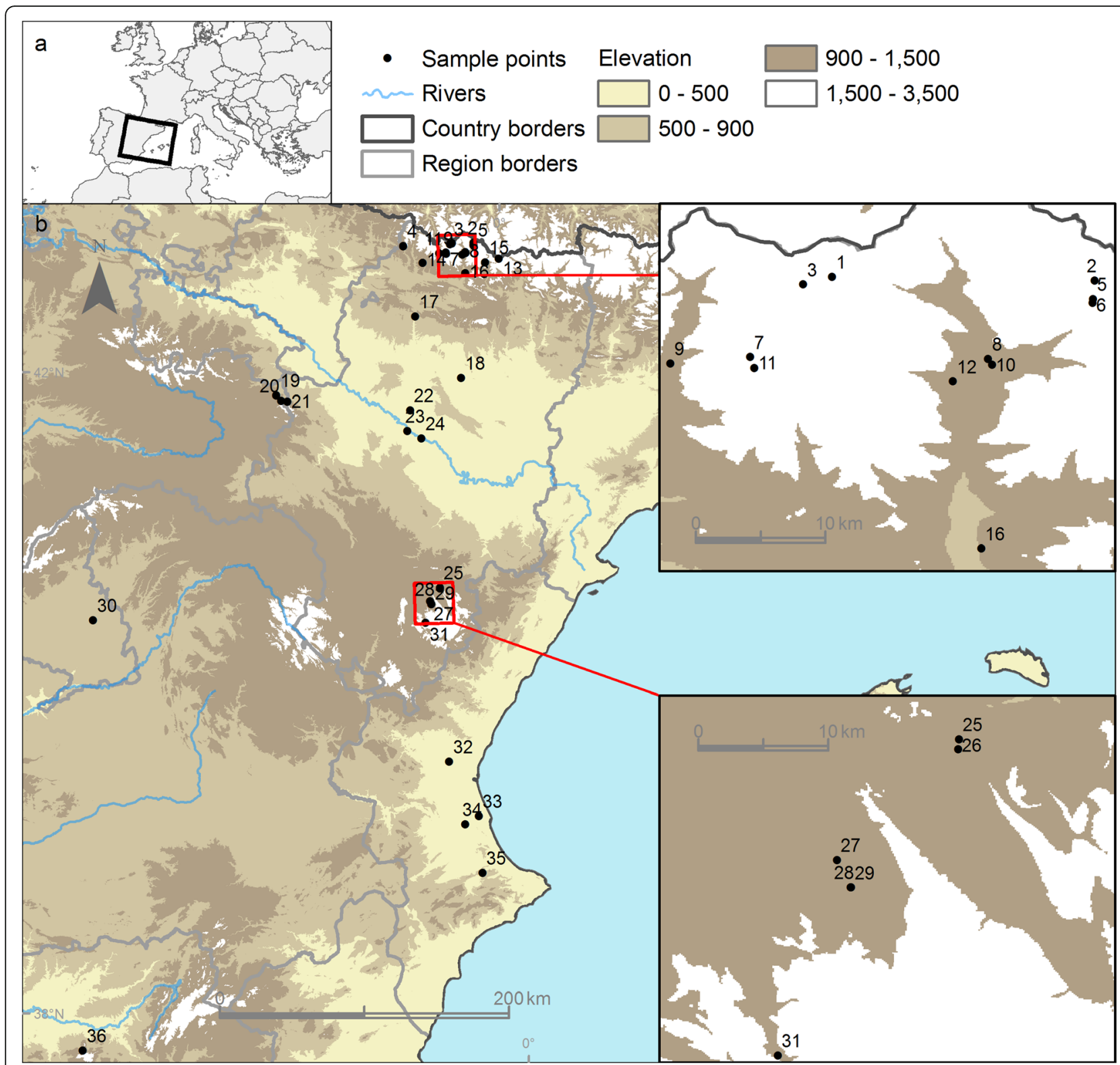

Fig. 1 Map of the different regions in Spain, showing the localities where blackflies were collected in this study. Localities are indicated by region: Aragón: 1-18, 21-29, 31; Castile and León: 19, 20; Madrid: 30; Valencia Community: 32-35; Andalusia: 36 (for full detail regarding the exact data of the localities, the reader is referred to the BOLD project: "DNA barcoding Spanish Blackflies [SPSIM, SPSIB, SIMSP]")

dataset also included cox 1 sequences of some species derived from UK blackflies analyzed in Day [10] and Day et al. [12] and retrieved from the GenBank database (Table 1). We analyzed the dataset in MEGA v.6 [33], and the neighbour-joining (NJ) analysis was undertaken using the K2P distance metric to represent species distribution pattern in the NJ tree. The robustness of the NJ tree was calculated using the bootstrap methodology employing 1000 as pseudoreplicates; only groups with $70 \%$ support values were mapped in the NJ tree as suggested [33]. Barcodes longer than $500 \mathrm{bp}$ were allocated a barcode index number (BIN) [34]. Each BIN was then mapped onto the NJ tree to examine their distribution among morphologically identified species. We also used the analyses capabilities of BOLD to determine the taxonomic incongruence between species identified by morphology and the cox1 DNA barcoding sequence [34].

\section{Results}

A total of 21 morphospecies of blackflies from Spain were included in the analyses: four of the genus Prosimulium and 17 of the genus Simulium, the latter belonging to the following subgenera: Boophthora (1 species); Eusimulium (1 species); Nevermannia (4 species); Simulium 
Table 1 List of the blackfly species, country of collection, number of specimens with DNA barcodes ( $n$ ) and mean intraspecific values of sequence divergence (\%)

\begin{tabular}{|c|c|c|c|}
\hline Species & Collection country & $n$ & Mean sequence divergence (\%) \\
\hline Prosimulium hirtipes & Spain, UK & 11 & 0.62 \\
\hline Prosimulium latimucro (s.l. $)^{a}$ & Spain, UK & 5 & 2.93 \\
\hline Prosimulium rufipes (s.l.) & Spain & 4 & 0.82 \\
\hline Prosimulium tomosvaryi ${ }^{\mathrm{a}}$ & Spain & 17 & 2.77 \\
\hline Simulium (Boophthora) erythrocephalum & Spain & 13 & 0.42 \\
\hline Simulium (Eusimulium) angustipes & UK & 9 & 1.09 \\
\hline Simulium (Eusimulium) petricolum & UK & 5 & 1.66 \\
\hline Simulium (Eusimulium) rubzovianum ${ }^{\text {b }}$ & Spain, UK & 16 & 1.63 \\
\hline Simulium (Nevermannia) bertrandi & Spain & 10 & $n a^{c}$ \\
\hline Simulium (Nevermannia) brevidens & Spain & 7 & 0.05 \\
\hline Simulium (Nevermannia) carthusiense & Spain & 10 & 0.35 \\
\hline Simulium (Nevermannia) cryophilum (s.l.) & Spain & 7 & 0.81 \\
\hline Simulium (Nevermannia) vernum (s.l.) & Spain, UK & 11 & 0.71 \\
\hline Simulium (Simulium) intermedium ${ }^{a}$ & Spain & 21 & 3.96 \\
\hline Simulium (Simulium) ornatum (s.I.) & Spain & 13 & 1.62 \\
\hline Simulium (Simulium) argyreatum ${ }^{a}$ & Spain, UK & 17 & 2.71 \\
\hline Simulium (Simulium) monticola & Spain & 14 & 0.45 \\
\hline Simulium (Simulium) variegatum ${ }^{\mathrm{a}}$ & Spain, UK & 22 & 2.15 \\
\hline Simulium (Simulium) xanthinum & Spain & 1 & $n a^{c}$ \\
\hline Simulium (Trichodagmia) galloprovinciale & Spain & 2 & 0.17 \\
\hline Simulium (Wilhelmia) equinum & Spain & 5 & 0.56 \\
\hline Simulium (Wilhelmia) lineatum & Spain & 8 & 1.11 \\
\hline Simulium (Wilhelmia) pseudequinum & Spain, UK & 6 & 1.19 \\
\hline Simulium (Wilhelmia) sergenti & Spain & 15 & 0.35 \\
\hline
\end{tabular}

${ }^{a}$ Taxa with high level of genetic diversity

${ }^{\mathrm{b}}$ The name of S. rubzovianum replaced S. velutinum for previous distributional records of this species in Spain, which is now restricted to North Africa and Canary Islands [43]

${ }^{C}$ Mean intraspecific values of sequence divergence (K2P) shown with missing entries (na) indicate that less than two specimens were analyzed

(s.s) (6 species); Trichodagmia (1 species); and Wilhelmia (4 species) (Table 1, Fig. 2). Additionally, S. (Nevermannia) bertrandi was identified only morphologically, but it was not possible to obtain DNA from the single specimen we collected. Three or more representatives were available for 21 morphospecies except for $S$. xanthinum and S. galloprovinciale (Table 1). In total, 239 individuals were analyzed after incorporating cox 1 sequences for certain species in Day [10] and Day et al. [12]. Of these, 225 yielded barcodes of full length 658 bp (94.14\% success).

The sequence divergences varied across all taxa we analyzed. For those individuals of the same species found in the same locality the genetic diversity was zero or ranked below average values, e.g. S. brevidens (see Table 1). On the contrary, other individuals revealed higher values, for example $P$. tomosvaryi (Table 1 ). The average for the intraspecific divergence was $1.47 \%$ (range $0.05-3.96 \%$ ) (see
Table 1), and the average for interspecific divergence ranged between 2.50-22.0\% (see Additional file 1: Table S1). All taxa belonging to separate genera (or subgenera) [1], showed increased values of genetic divergence. For example, the most divergent pairs were $P$. tomosvaryi/S. equinum (22.0\%) and P. latimucro (s.l.)/S. equinum, $P$. latimucro (s.l.)/S. monticola, $P$. tomosvaryi/S. brevidens, $S$. equinum/P. hirtipes, S. brevidens/P. hirtipes and S. xanthinum/P. hirtipes (21.0\%). As expected, species within the same genus or subgenus showed low genetic divergence, for example S. brevidens/S. cryophilum (s.l.) (2.5\%).

In general, individuals belonging to the same species clustered together including those specimens that were collected far apart. However, not all morphospecies displayed a similar pattern (see Fig. 2). In this case, specimens recognized as $S$. argyreatum, S. monticola and $S$. variegatum assembled together in the same group with high bootstrap values (Fig. 2). 


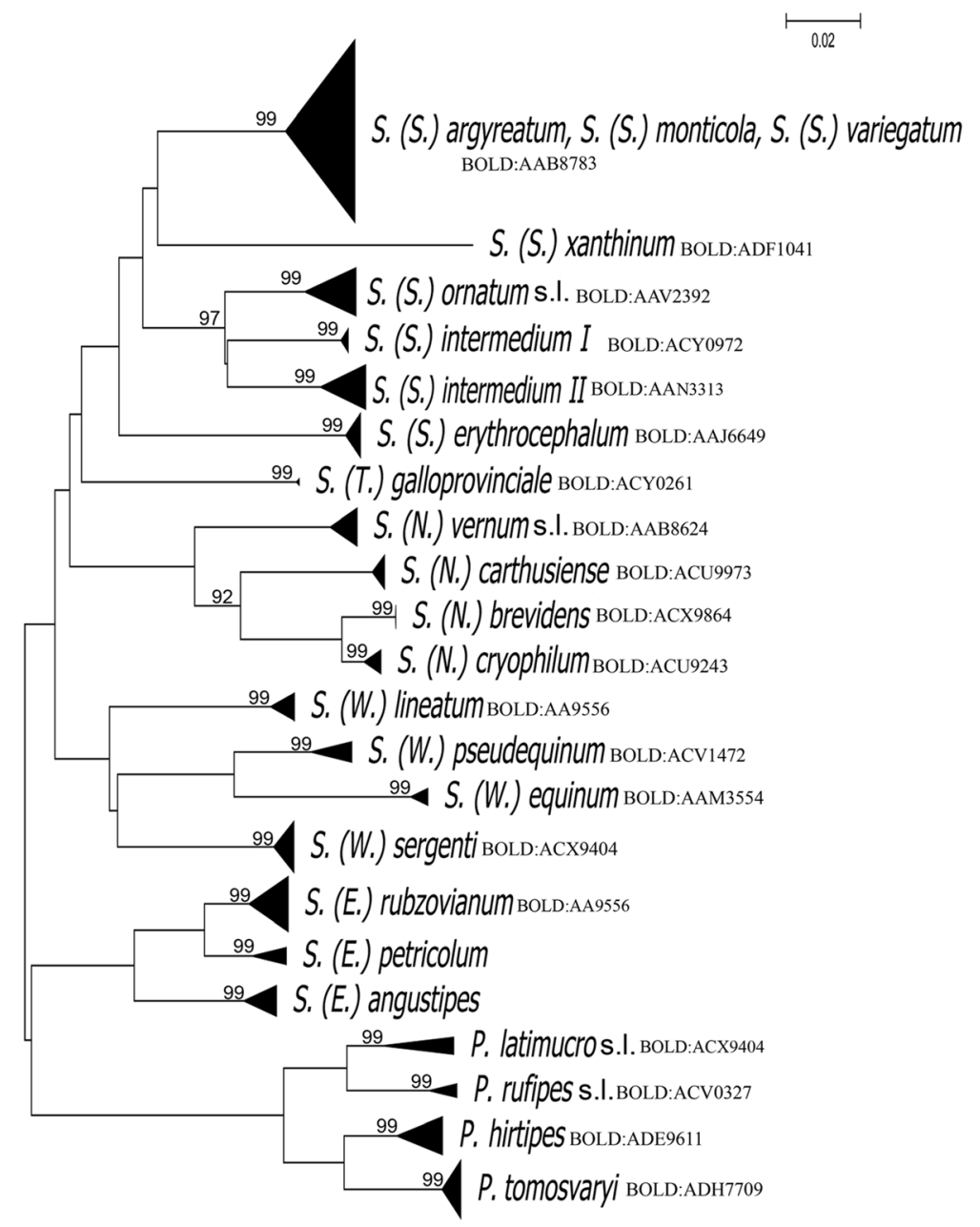

Fig. 2 Neighbour-joining tree for species of the Simuliidae sampled in Spain based on 658 bp barcodes from the cox 1 DNA barcoding region. Only bootstrap values higher than $70 \%$ are shown

In this study, five species are known species complexes [1]: P. latimucro (s.l.), P. rufipes (s.l.), S. ornatum (s.l.), S. cryophilum (s.l.) and S. vernum (s.l.).

The BIN calculation in the dataset of 171 barcode records was of $21 \mathrm{BINs}$, which were representatives of 21 species. Our BIN count did not include sequences from Day [10] and Day et al. [12]. In general, 133 barcodes were assigned a BIN number, which represented 16 concordant BINs, four singletons, and one discordant BIN (33 records). The discordant BIN was discordant at the species level S. argyreatum, S. monticola and S. variegatum (BIN AAB8783). In contrast, BIN splits were detected in P. latimucro (s.l.) (BINs ADE9519, ACX9973) and S. intermedium (BINs AAN3313, AAV2392) (Fig. 2).

\section{Discussion}

Recent arguments on the utility of cox1 DNA barcoding in blackflies have been discussed by [22-24, 35]. In our study, known species clustered together in the NJ tree based upon cox1 DNA barcode sequences (Fig. 2), which demonstrate the utility of this methodology in support of species identification. Most of the individuals of a given species were correctly placed in the NJ tree. Nonetheless, morphological specimens identified as $S$. argyreatum, S. monticola and S. variegatum were mapped in the same cluster, implying that they might be conspecific. This result was not surprising as the adults of the three species are morphologically very similar. However, the three taxa can be readily identified based on the pupal gill configuration. Simulium variegatum is easily identified by having $1+1$ prominent tubercles at the base of the gill [26], while the tubercles are absent in S. argyreatum and S. monticola. In S. monticola, the ventral gill filaments originate directly from the base, all filaments are prominently curved at mid length, and the cephalothorax is covered by areas of small tubercles [26]. In $S$. argyreatum, the gill is covered by tubercles, which are homogeneously distributed [26]. Thus, we advocate that 
different genetic markers such as the elongator complex protein 1 gene (ECP1) or ITS2 [36-39] should be used to explore their taxonomic status.

We expected higher levels of genetic variation between members of known species complexes, even though cytological studies were not carried out in our study [3, 22-24, 40]. With this regard, most of the specimens grouped together, and high levels of genetic diversity was not identified between species complexes. In addition, no deep divisions in the NJ tree as observed in previous studies [3, $22,24]$. This is likely due to the fact that most of the specimens originated from the same, or relatively close, localities. However, not all known species grouped as we anticipated. As a whole, we revealed high intraspecific genetic divergence not only in P. latimucro (s.l.) with $2.77 \%$, but also in P. tomosvaryi with $2.93 \%$ and $S$. intermedium with $3.96 \%$. In particular, S. intermedium was split into two distinct groups, named here I and II (Fig. 2). This could be indicative of the presence of a species complex, but further cytotaxonomic studies are required to validate this hypothesis. In this study, the values obtained for the intraspecific genetic divergences as well as for the interspecific genetic divergences are within the values obtained by other authors [22-24, 40-42].

Many authors (e.g. [34, 35]) have stated that the congruence found between morphologically recognized species and BINs could demonstrate the presence of cryptic genetic diversity. Therefore, the subgroups detected in $S$. intermedium may be indicative of such diversity. In contrast, the presence of same BINs in other recognized species such as S. argyreatum, S. monticola and S. variegatum, might be hard to explain. Therefore, we advocate for further biosystematic studies in these taxa not only in Spain, but across their distribution range.

\section{Conclusions}

Our study augments the available information with regards to the utility of the cox 1 DNA barcoding to assist the identification in the understudied Spanish blackfly fauna. Our results emphasize the need for continuing research using an integrated research approach using a combination of morphological traits and molecular markers, not only on the Spanish Simuliidae fauna, but also across the world. This comprehensive approach would support the correct identification of blackfly species, which in turn would have a direct effect for the implementation of the correct vector control strategies or the development of accurate protocols for studies in disease dissemination.

\section{Additional file}

Additional file 1: Table S1. Interspecific (between groups) pairwise K2P genetic divergence of unique DNA barcodes, representing 23 species of the Simuliidae. (DOCX $25 \mathrm{~kb}$ )

\section{Abbreviations}

BOLD: Barcode of Life Database; BIN: barcode index number;

cox1: cytochrome $c$ oxidase subunit 1; ECP1: Elongator complex protein 1; NJ tree: neighbour-joining tree

\section{Acknowledgements}

The authors would like to acknowledge the authorities of INAGA from the Government of Aragon for providing the permit to conduct the fieldwork in Aragón region. Thanks are also given to Dr David Martínez and Paloma Pik for their help in field collection and Dr Antonio Ortiz for sending samples from Jaen. We thank Dr Pablo Miguel Lucas for his help with Fig. 1. Thanks are given to the Sequencing Unit at APHA, especially Jeremy Hawthorn, Victoria Rose and Steve Shankster for their efforts in obtaining good quality DNA sequences for this project. Partial results from this study were presented at the VII International Simuliidae Symposium held in Zaragoza (Spain) in September 2016 and at the 7th SOVE International Congress held in Mallorca (Spain) in October 2017.

\section{Funding}

This work was funded by the Department for Environment, Food and Rural Affairs (DEFRA), Scottish Government and Welsh Government through grants SV3045, and SE4113. The study was supported by the Ministry of Education, Science and Technological Development of the Republic of Serbia (projects III43007 and TR31084) and by the Consejo Nacional de Ciencia y Tecnología of México (MEXBOL Red de Códigos de Barras de la Vida, Grant No. 271108).

\section{Availability of data and materials}

The data used in this study are included in this published article. The newly generated sequences were submitted to the GenBank database under the accession numbers MG894170-MG894340. Additional information can be requested from the corresponding author.

\section{Authors' contributions}

IRA, JLC and LMHT designed the study. IRA, JAGH, MARP and LMHT conducted the surveys. IRA and AIC conducted the morphological identification. LMHT performed the molecular analysis. NN performed the alignment of the sequences. IRA, LMHT, JAO and ARF analyzed the results. IRA and LMHT compiled the main information and wrote the manuscript. All authors critically reviewed, read and approved the final manuscript.

Ethics approval and consent to participate

Not applicable.

Consent for publication

Not applicable.

Competing interests

The authors declare that they have no competing interests.

\section{Publisher's Note}

Springer Nature remains neutral with regard to jurisdictional claims in published maps and institutional affiliations.

\footnotetext{
Author details

${ }^{1}$ Center for Rickettsiosis and Arthropod-Borne Diseases, Hospital Universitario San Pedro-CIBIR, Logroño, La Rioja, Spain. ${ }^{2}$ Department of Animal Pathology, Faculty of Veterinary Sciences, Universidad de Zaragoza, Zaragoza, Spain. ${ }^{3}$ Wildlife Zoonoses and Vector-borne Diseases Research Group, Virology Department, Animal and Plant Health Agency, Addlestone, UK. ${ }^{4}$ Faculty of Agriculture, University of Novi Sad, Novi Sad, Vojvodina Province, Serbia. ${ }^{5}$ University of Liverpool, Liverpool, UK. ${ }^{6}$ Instituto de Ciencias Biomédicas, Universidad Autónoma de Ciudad Juárez, Ciudad Juárez, Chihuahua, México. ${ }^{7}$ Centro de Biotecnología Genómica, Instituto Politécnico Nacional, Reynosa, Tamaulipas, México. ${ }^{8}$ Department of Clinical Infection, Microbiology and Immunology, Institute of Infection and Global Health, University of Liverpool, Liverpool, UK.
} 
Received: 11 May 2018 Accepted: 1 August 2018

\section{Published online: 13 August 2018}

\section{References}

1. Adler PH, Crosskey RW. World blackflies (Diptera: Simuliidae): a comprehensive revision of the taxonomic and geographical inventory. 2018. https://biomia.sites.clemson.edu/pdfs/blackflyinventory.pdf. Accessed $14 \mathrm{Mar}$ 2018.

2. Shelley AJ, Hernández LM, Maia-Herzog M, Luna Dias APA. Garritano PR. In: Adis J, Arias J, Golovatch S, Mantzev KM, Rueda-Delgado G, Domínguez E, editors. Aquatic Biodiversity in Latin America ABLA Series, vol. 6. SofiaMoscow: Pensoft Publishers; 2010

3. Hernández-Triana LM. Montes de Oca F, Prosser SW, Hebert PD, Gregory RT, McMurtrie S. DNA barcoding as an aid for species identification in austral black flies (Insecta: Diptera: Simuliidae). Genome. 2017;60:348-57.

4. Colebunders R, Hendy A, Nanyunja M, Wamala JF, Van Oijen M. Nodding syndrome - a new hypothesis and new direction for research. Int J Inf Dis. 2014:27:74-7.

5. Malmquivist B, Adler PH, Kuusela K, Merritt RW, Wotton RS. Suspension feeders transform massive amounts of seston in large northern rivers. Oikos. 2001;92:35-43

6. Malmquivist B, Adler PH, Kuusela K, Merritt RW, Wotton RS. Black flies in the boreal biome, key organisms in both terrestrial and aquatic environments: a review. Écoscience. 2004;11:187-200.

7. Feld CK, Kiel E, Lautenschläger M. The indication of morphological degradation of streams and rivers using Simuliidae. Limnologica. 2002;32: 273-88

8. Currie DC, Adler PH. Global diversity of black flies (Diptera: Simuliidae) in freshwater. Hydrobiologia. 2008;595:469-75.

9. Pramual P, Kuvangkadilok C. Agricultural land use and blackflies (Diptera, Simuliidae) species richness and species assemblages in tropical streams, northern Thailand. Hidrobiologia. 2009;625:173-84.

10. Day JC. Using molecular tools to differentiate closely related blackfly species of the genus Simulium. Bristol: Environment Agency; 2008.

11. Day JC, Goodall TI, Post RJ. Confirmation of the species status of the blackfly Simulium galeratum in Britain using molecular taxonomy. Med Vet Entomol. 2008;22:55-61.

12. Day JC, Mustapha M, Post RJ. The subgenus Eusimulium (Diptera: Simuliidae: Simulium) in Britain. Aquat Ins. 2010;34:281-91.

13. Ilmonen J, Adler PH, Malmqvist B, Cywinska A. The Simulium vernum group (Diptera: Simuliidae) in Europe: multiple character sets for assessing species status. Linn Soc Lon. 2009;156:847-53.

14. Kúdela M, Brúderová T, Jedlička L, Bernotienè R, Celec P, Szemes T. The identity and genetic characterization of Simulium reptans (Diptera: Simuliidae) from central and northern Europe. Zootaxa. 2014;3802:301-17.

15. Kúdela M, Adler PH, Kúdelová T. Taxonomic status of the black fly Prosimulium italicum Rivosecchi (Diptera: Simuliidae) based on genetic evidence. Zootaxa. 2018:4377:280-90.

16. Adler PH, Kúdelová T, Kúdela M, Seitz G, Ignjatović-Ćupina A. Cryptic biodiversity and the origins of pest status revealed in the macrogenome of Simulium colombaschense (Diptera: Simuliidae), history's most destructive black fly. PLoS One. 2016;11:e0147673.

17. Ruiz-Arrondo I, Alarcón-Elbal PM, Figueras L, Delacour-Estrella S, Muñoz A, Kotter $\mathrm{H}$, et al. Expansión de los simúlidos (Diptera: Simuliidae) en España: un nuevo reto para la salud pública y la sanidad animal. Bol SEA. 2014;54: 193-200.

18. Sánchez-López PF, Ruiz-Arrondo I, Kotter H, Pacheco Martínez F, Segovia Hernández M, Gómez Campoy ME. Nuevos retos en gestión de vectores en salud pública: la mosca negra en Murcia (España). Gac Sanit. 2018;32:181-3.

19. Figueras L, Lucientes J, Ruiz I, Ramos JJ, Ferrer LJ. Caso clínico: ataque de simúlidos en rumiantes. Albéitar. 2011;147:22-3.

20. Ruiz-Arrondo I, Garza-Hernández JA, Reyes-Villanueva F, Lucientes-Curdi J, Rodríguez-Pérez MA. Human-landing rate, gonotrophic cycle length, survivorship, and public health importance of Simulium erythrocephalum in Zaragoza, northeastern Spain. Parasit Vectors. 2017;10:175.

21. Cywinska A, Hunter FF, Hebert PDN. Identifying Canadian mosquitoes species through DNA barcodes. Med Vet Entomol. 2006;20:413-24.

22. Hernández-Triana LM, Crainey JL, Hall A, Fatih F, Mackenzie-Dodds J, Shelley AJ, et al. The utility of DNA barcoding for species identification within the blackfly subgenus Trichodagmia Enderlein (Diptera: Simuliidae: Simulium) and related taxa in the New World. Zootaxa. 2012;3514:43-69.
23. Hernández-Triana LM, Prosser SW, Rodríguez-Pérez MA, Chaverri LG, Hebert PDN, Gregory RT. Recovery of DNA barcodes from blackfly museum specimens (Diptera: Simuliidae) using primer sets that target a variety of sequence length. Mol Ecol Res. 2014;14:508-18.

24. Hernández-Triana LM, Prosser SW, Rodríguez-Pérez MA, Chaverri LG, Hebert PDN, Gregory RT, et al. DNA barcoding Neotropical black flies (Diptera: Simuliidae): species identification and discovery of cryptic diversity in Mesoamerica. Zootaxa. 2015;3936:93-114.

25. Hernández-Triana LM. Recommendations by The Natural History Museum on methods for collecting, preserving, rearing and mailing of simuliid specimens. 2007. Available from http://blackflies.info/content/simuliidaecollecting-protocol. Accessed 12 July 2014.

26. González G. Claves para la identificación de las larvas y pupas de los Simulidos (Diptera) de la Península Ibérica. Barcelona: Asociación Española de Limnología; 1997.

27. Bass JAB. Last-instar larvae and pupae of the Simuliidae of Britain and Ireland. A key with brief ecological notes. Freshwater Biological Association, Scientific Publication No. 55. Ambleside: Freshwater Biological Association; 1998.

28. Rivosecchi L, Addonisio M, Maiolini B. I Ditteri Simulidi. Nuove chiavi dicotomiche per l' identificazione delle specie italiane con brevi note bio-tassonomiche. Trento: Quaderni del museo Tridentino di Scienze naturali, 2; 2007.

29. Montero-Pau J, Gómez A, Muñoz J. Application of an inexpensive and high throughput genomic DNA extraction method for the molecular ecology of zooplanktonic diapausing eggs. Limnol Oceanogr Methods. 2008;6:218-22.

30. Folmer O, Black M, Hoeh W, Lutz R, Vrijenhoek R. DNA primers for amplification of mitochondrial cytochrome oxidase subunit I from diverse metazoan invertebrates. Mol Mar Biol Biotechnol. 1994;3:294-9.

31. Hebert PDN, Cywinska A, Ball SL, DeWaard JR. Biological identifications through DNA barcodes. Proc R Soc B Sc. 2003;270:313-21.

32. Hebert PDN, Ratnasingham S, DeWaard JR. Barcoding animal life: cytochrome $c$ oxidase subunit 1 divergences among closely related species. Proc R Soc B Sc. 2003;270:S96-9.

33. Tamura K, Stecher G, Peterson D, Filipski A, Kumar S. MEGA6: Molecular Evolutionary Genetics Analysis version 6.0. Mol Biol Evol. 2013;30:2725-9.

34. Ratnasingham $\mathrm{S}$, Hebert PND. A DNA-based registry for all animal species: the Barcode Index Number (BIN) System. PLoS One. 2013;8:e66213.

35. Hernández-Triana LM. Systematics of the blackfly subgenus Trichodagmia Enderlein (Diptera: Simuliidae: Simulium) in the New World. PhD thesis, Wageningen University, Wageningen, Netherlands; 2011.

36. Senatore GL, Alexander EA, Adler PH, Moulton JK. Molecular systematics of the Simulium jenningsi species group (Diptera: Simuliidae), with three new fast-evolving nuclear genes for phylogenetic inference. Mol Phyl Evol. 2014; 75:138-48.

37. Low VL, Pramual P, Adler PH, Ya'cob Z, Huang YT, Da Pham X, et al. Delineating taxonomic boundaries in the largest species complex of black flies (Simuliidae) in the Oriental Region. Sci Rep. 2016;6:20346.

38. Pangjanda S, Pramual P. Tests of conspecificity for closely related black fly (Diptera: Simuliidae) species of Simulium multistriatum group in Thailand. Zootaxa. 2017:4231:Zootaxa.4231.3.8.

39. Thaikarern J, Pramual P, Adler P. Life-stage association of black flies, using a fast-evolving nuclear gene sequence, and description of the larva of Simulium lampangense Takaoka \& Choochote (Diptera: Simuliidae) from Thailand. Zootaxa. 2017;4299:zootaxa.4299.2.6

40. Rivera J, Currie D. Identification of Nearctic blackflies using DNA barcodes (Diptera: Simuliidae). Mol Ecol Res. 2009:9:224-36.

41. Pramual P, Adler PH. DNA barcoding of tropical black flies (Diptera: Simuliidae) of Thailand. Mol Ecol Res. 2014;14:262-71.

42. Pramual P, Thaijarem J, Wongpakam K. DNA barcoding of human-biting black flies (Diptera: Simuliidae) in Thailand. Acta Trop. 2016;164:33-40.

43. Adler PH, Cherairia M, Arigue SF, Samraou BI, Belqat B. Cryptic biodiversity in the cytogenome of bird-biting blackflies in North Africa. Med Vet Entomol. 2015:29:276-89. 